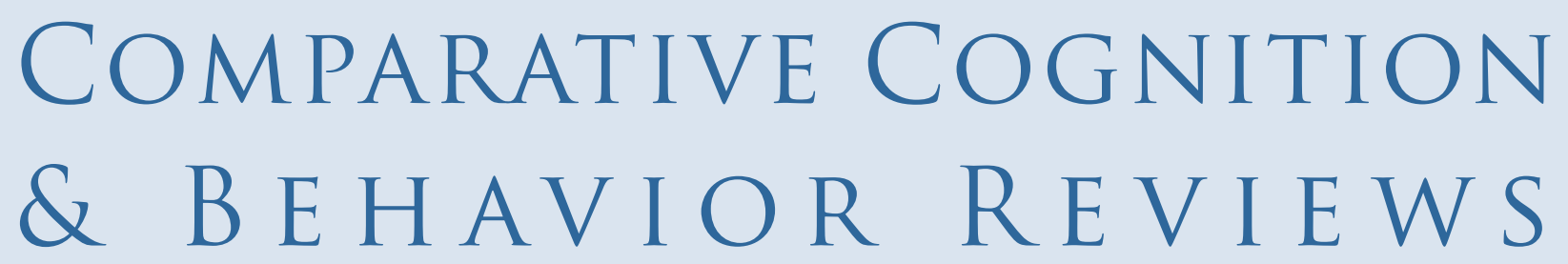

\title{
Animal Pitch Perception: Melodies and Harmonies
}

\author{
Marisa Hoeschele \\ University of Vienna
}

Pitch is a percept of sound that is based in part on fundamental frequency. Although pitch can be defined in a way that is clearly separable from other aspects of musical sounds, such as timbre, the perception of pitch is not a simple topic. Despite this, studying pitch separately from other aspects of sound has led to some interesting conclusions about how humans and other animals process acoustic signals. It turns out that pitch perception in humans is based on an assessment of pitch height, pitch chroma, relative pitch, and grouping principles. How pitch is broken down depends largely on the context. Most, if not all, of these principles appear to also be used by other species, but when and how accurately they are used varies across species and context. Studying how other animals compare to humans in their pitch abilities is partially a reevaluation of what we know about humans by considering ourselves in a biological context.

\section{Keywords: pitch, music, acoustics, perception}

\section{Introduction}

Music is found in all human cultures and, much like language, there are universals in musical systems across cultures (Brown \& Jordania, 2011). Because music appears to be part of what it means to be human, it follows that researchers have become interested in the biology of music: What is it about human biology that causes us to have music (Fitch, 2006; Patel, 2003)? One way to shed light on this question is to compare humans to other species. Comparative studies have suggested that many abilities found in humans that relate to our passion for music are not unique to our species (Hoeschele, Merchant, Kikuchi, Hattori, \& ten Cate, 2015).

Because human music is also influenced greatly by the general human trait of complex cultural evolution, sometimes the complexity of present-day music is taken as a sign that human music is quite unlike anything that other species produce. However, the universals we find across cultures, such as discrete pitches and the use of pitch intervals with simple interval ratios (Brown \& Jordania, 2011; Burns, 1981; Carterette \& Kendall, 1999), are sometimes also found in isolation in other species (e.g., Cator, Arthur, Harrington, \& Hoy, 2009; Doolittle \& Brumm, 2013; Doolittle, Gingras, Endres, \& Fitch, 2014). Because pitch plays a central role in the discussion of human music universals, it is the focus of the current special issue on animal music perception (see preface in this issue for an introduction).

Pitch is a percept of sound that is correlated with fundamental frequency (Dowling \& Harwood, 1986). When we describe human female voices as being "higher" than male voices, or a melody going "up" and "down," we are talking about changes in pitch. Pitch is a very important attribute of sound that carries information in human speech, human music, and the vocalizations of many nonhuman species (Doupe \& Kuhl, 1999; Rothenberg, Roeske, Voss, Naguib, \& Tchernichovski, 
2014). Pitch information transmitted by animal species is sometimes based in physical acoustics. For example, larger objects tend to make lower pitched sounds, and animals have been shown to exploit this fact in order to sound larger (Fitch \& Hauser, 2003). Pitch information can also be learned, such as the role of pitch the Vietnamese word mott, which can mean either "trendy" or "one" based on the pitch inflection (Kim et al., 2016).

It is important that we disambiguate pitch from timbre, both of which are based on the spectral properties of a sound. Whereas pitch is correlated with the fundamental frequency of a sound, timbre is derived from the rest of the spectral information (Dowling \& Harwood, 1986). Timbre gives sound its quality, for example, the difference between the same note played on the piano and on the violin. A piano and a violin following the same note pattern on sheet music are playing the same pitches but not the same timbre. As one can see from this example, in music pitch and timbre are clearly separated. The notes written on sheet music are pitch, whereas the expression and instrumentation of this sheet music is the timbre.

Because of this definitional distinction between pitch and timbre, it is often taken for granted that humans also perceive these two aspects of sound as distinct. In some sense, this is true. For example, the tune "Happy Birthday" is recognizable whether it's being hummed or whether it's being played by a violin. As such, timbre has been referred to as a "surface feature" rather than a deeper structural feature of music (e.g., Halpern \& Müllensiefen, 2008; Schellenberg, Stanlinski, \& Marks, 2013; Warker \& Halpern, 2005). However, we know that timbre contributes to how pitch information is processed (Lange \& Czernochowski, 2013; Weiss, Vanzella, Schellenberg, \& Trehub, 2015). In addition, it is so difficult to extract pitch information from a recording of modern music that there is so far no trusted algorithm to do so (Benetos, Dixon, Giannoulis, \& Kirchhoff,

Author Note: Marisa Hoeschele, Department of Cognitive Biology, University of Vienna, Vienna, Austria.

Correspondence concerning this article should be addressed to Marisa Hoeschele at marisa.hoeschele@univie.ac.at

Acknowledgments: Marisa Hoeschele was funded by a Lise Meitner Postdoctoral Fellowship (M 1732-B19) from the Austrian Science Fund (FWF) during the writing of this manuscript. This paper is part of a special issue that is based on a symposium organized by the author entitled "Evolution of Music" at the Comparative Cognition Conference in March 2016.
2013). Instead, researchers analyzing pitch in music rely heavily on transcriptions of the music into musical notation by expert human listeners, such as the McGill billboard project (Burgoyne, Wild, \& Fijinaga, 2011). As pitch transcription in songs that have many instruments is extremely difficult and sometimes even impossible for expert listeners (Klapuri \& Davy, 2006), it is very unlikely that the average human listener can always clearly separate pitch and timbre in the music they encounter.

Despite this, focusing on pitch in isolation is a good starting place to begin to understand how pitch information is used across species. Outside of the complex music we listen to today, the individual vocal signals we encounter have much simpler pitch information. In speech, pitch alone can change both emotional and semantic information (Bowling, Gill, Choi, Prinz, \& Purves, 2010; Curtis \& Bharucha, 2010; Filippi, 2016). In addition, there is evidence that both mammalian and avian species perceive fundamental frequency (the acoustic correlate of pitch) even if it has been removed from the signal (Cynx \& Shapiro, 1986; Heffner \& Whitfield, 1976; Tomlinson \& Schwarz, 1988), an ability already present in human infants (Lau \& Werner, 2012). This ability to break down acoustic stimuli to assess fundamental frequency allows animals to parse pitch information even in signals where the lower frequencies are lost or masked. A common example of this ability being put to use is found in humans: We are able to recognize the low pitch of a male voice over the phone, even though the lower frequencies that distinguish the male voice from female have been lost in the transmission process.

Another reason to study pitch separately from timbre is that pitch perception is quite complicated, even in humans. From our initial description just presented, one might imagine that pitch perception is simply the ability to identify a $220 \mathrm{~Hz}$ tone as $220 \mathrm{~Hz}$ and a $440 \mathrm{~Hz}$ tone as $440 \mathrm{~Hz}$. However, humans are typically quite poor at this. Instead, we might treat these two notes as the same note, because they are harmonically related. Harmonics are overtones that occur at integer multiples of the fundamental frequency. Because $440 \mathrm{~Hz}$ is an integer multiple of $220 \mathrm{~Hz}(2 \times)$ it is also a naturally occurring harmonic of $220 \mathrm{~Hz}$. We also might assess two notes in a relative way: $440 \mathrm{~Hz}$ is higher than $220 \mathrm{~Hz}$ by 12 semitones, which sounds similar to notes separated by a distance of 11 or 13 semitones. Relative assessments can get more complicated still: We might say the ratio of 220 to $440 \mathrm{~Hz}$ is 1:2, a simple ratio, which sounds similar to other simple ratios such as $2: 3$, which are only seven 
semitones apart. With all these different ways of assessing pitch, there is no simple answer as to how a pattern of notes will be perceived even in our own species. Next I discuss each of these methods of assessing pitch in more depth and outline what we know about each of them across species. Finally, I discuss where the combined results suggest we should go in future research.

\section{Absolute Pitch \\ What Is Absolute Pitch?}

In music, absolute pitch (also known as "perfect pitch") refers to the ability to identify a musical note without an external reference, for example, identifying $220 \mathrm{~Hz}$ or $440 \mathrm{~Hz}$ as an "A" note. This is a rare ability that has been said to occur in as few as one in 10,000 people (Bachem, 1955). People are typically defined as either having absolute pitch or not having it. When we are just discussing our own species, separating humans into these two groups makes a lot of sense because the ability does appear to be mostly categorical, not continuous (Athos et al., 2007), at least when it comes to generalization across timbres (Baharloo, Johnston, Service, Gitschier, \& Freimer, 1998; Lockhead \& Byrd, 1981).

However, the story changes when we consider our species in context with other species. Do people who lack musical absolute pitch as just described have no sense of absolute pitch at all? A quick thought experiment makes it clear that the answer is "no." Absolute pitch is the first piece of information we use to discriminate male and female voices (Latinus \& Taylor, 2012). And when asked to sing a popular recorded song, about two thirds of participants reproduced the absolute pitches from the recording fairly accurately (within two semitones) without prompting (Levitin, 1994). People are also quite good at identifying frequency shifted versions of a melody they know well (Schellenberg \& Trehub, 2003). Tones heard frequently, such as a dial tone on a home phone line, are also recognized at their normal pitch (Smith \& Schmuckler, 2008). The majority of humans are simply not accurate enough as absolute pitch assessors to label specific musical notes.

Pioneering work by Stewart Hulse and colleagues showed that several avian species appear to be quite different from humans in their perception of both absolute and relative pitch (Hulse, Bernard, \& Braaten, 1995; Hulse \& Cynx, 1985, 1986; Hulse, Cynx, \& Humpal, 1984; MacDougall-Shackleton \& Hulse, 1996; Page, Hulse, \& Cynx, 1989). In response to this work, Ron Weisman and many colleagues (Friedrich, Zentall, \& Weisman, 2007;
Lee, Charrier, Bloomfield, Weisman, \& Sturdy, 2006; Weisman et al., 2010; Weisman, Balkwill, Hoeschele, Moscicki, \& Sturdy, 2012) systematically assessed the absolute pitch abilities of humans and other mammalian and avian species when presented with tones using a simple operant conditioning task. The animals were rewarded for responding to some tones but not to other tones. The tones were divided into either three or eight frequency ranges of alternating reward contingency: For example, the lowest frequencies might be unrewarded, followed by a rewarded range, followed by unrewarded, and so on. Overall, the studies showed that the majority of humans and rats (Rattus norvegicus) were able to solve a three-range task but failed at an eight-range task, pigeons did a little better showing some success at the eight-range task, whereas several vocal learning bird species were able to solve both tasks with high accuracy (Weisman, Mewhort, Hoeschele, \& Sturdy, 2012).

The preceding data, with highly comparable methodology across species, makes the story we can tell about absolute pitch seem quite simple: Overall, mammals are less accurate relative to birds, especially birds that are vocal learners. However, it is difficult to draw such a sweeping conclusion from so few species, especially when we have not fully considered what we know about humans. What about those humans with musical absolute pitch abilities? Have we really told the whole story about humans relative to other species without taking these exceptions into account? It turns out humans with musical absolute pitch do very well at this task, and approach the level of discrimination that some of the birds had. However, they make some very striking errors, responding at chance level to some tones in the middle of a reinforced range (Weisman et al., 2010). These data made it clear that there was more to the absolute pitch story than we had initially anticipated.

\section{Pitch Height versus Pitch Chroma}

The initial work spearheaded by Ron Weisman focused on treating the perception of pitch much like how we assess most other stimuli that range in some continuous parameter such as size, brightness, loudness, and so forth: according to Weber's law. Weber's law is quite intuitive in many cases. If we compare a set of two objects to a set of three objects, it is easy to identify that the second set contains more objects. Whereas, if we compare a set of 100 objects to a set of 101 objects, it may be difficult to tell which set is larger even though both set comparisons involve adding one additional element. Pitch height is the perception of pitch according 
to Weber's law alone, where we perceive differences in frequency on a log linear scale.

Pitch chroma, by contrast, is what caused the humans with musical absolute pitch to make errors in the eight-range discrimination task, just described (Weisman et al., 2010). It is a circular way of perceiving pitch, where pitch repeats each time frequency doubles. For example, in Western music, if we begin on an A note on the piano and ascend the keyboard labeling the note of each key we pass, after 12 notes we have another A note and the pattern repeats. Each repetition is referred to as an "octave." In the range discrimination task, our participants treated tones that were double the frequency of other tones as the same tone. In other words, an A note was always an A note, regardless of its frequency. In cases where tones separated by an octave were reinforced differentially, participants were more likely to make errors. In other words, they were perceiving pitch in a circular fashion. Their absolute perception of pitch turned out to be more complicated than simply log linear. It repeated each time frequency doubled.

It appears that all humans, including those with musical absolute pitch, have a weak sense of pitch height. In the human range experiments, musical absolute pitch was identified in participants by a standardized test of musical absolute pitch (Athos et al., 2007). In the test, participants were required to name the note that they heard and indicate the octave that the note came from. Octaves were labeled with numbers 1 to 6 , and we reminded all participants that the fourth octave is where one can find "middle C" on the piano. We found that when it came to labeling what octave a note came from, participants with absolute pitch were no better than participants without absolute pitch (Weisman et al., 2010). It turns out that this is a commonly observed phenomenon: Humans with musical absolute pitch do not have better pitch height judgments than those without musical absolute pitch (Carroll, 1975; Deutsch \& Henthorn, 2004; Lockhead \& Byrd, 1981; Miyazaki, 1988, 1989; Takeuchi \& Hulse, 1993).

Now it was clear that all humans share a roughly similar ability to assess pitch height, regardless of whether they had musical absolute pitch. People with musical absolute pitch relied on their perception of pitch chroma to solve an absolute pitch task designed to assess pitch height perception. Our next question concerned human participants without musical absolute pitch: Did they also hear pitch chroma and were just not as accurate at identifying it? There is some evidence that all humans attend to pitch chroma. Given how poor humans are at pitch height but how well humans do at recognizing and producing familiar melodies and notes at their normal pitch (Levitin, 1994; Schellenberg \& Trehub, 2003; Smith \& Schmuckler, 2008), it is possible that they are using pitch chroma to solve these tasks. Humans also appear to remember pitch chroma based on how their own voice and dialect make use of it (Deutsch, 1991). It seems that all humans have some level of implicit pitch chroma perception, but it does not typically present itself outside of context. How, then, can we study it in other species?

Before turning to other species, it is important to discuss pitch chroma in more detail, because it otherwise may sound like an arbitrary phenomenon that we should not expect to find in other species. Pitches with the same chroma but different pitch height are separated by one or more octaves. A note and its octave have an integer ratio of $1: 2$, the simplest ratio outside of unison (1:1). Octaves are found in the harmonics of natural vocalizations. The harmonics found in vocalizations are integer multiples of the fundamental frequency. Because of this, the relationship between the first harmonic of an acoustic signal and its fundamental frequency is always an octave. Many of the other harmonics also have octave relationships to the fundamental frequency. See Figure 1 for a visual depiction of this information. It is common in the human literature to talk about how the octave is "special" and how humans treat notes separated by an octave as being the same. In some sense it is clear that this is the case. When humans with different vocal ranges sing together, such as a child and his or her father, they are said to be singing the "same thing" when they match chroma even if they are singing in different octaves. This may seem like an arbitrary convention, but in fact it makes a lot of sense when one considers the acoustic signal. On average, human male and female voices are roughly an octave apart (Titze, 2000). It is therefore natural to produce vocalizations separated by an octave. Also, because the octave has a simple acoustic relationship naturally found within harmonics, octave transposition of the fundamental frequency produces the closest harmonic match to the original signal (other than reproducing the same fundamental frequency; see Figure 1). As an imitating species in which individuals have different vocal ranges, it makes a lot of sense for humans to utilize the properties of the octave to approximate the vocal sound they are trying to imitate. This separation in vocal range among humans may thus underlie why the octave is "special" in our species.

Even though the octave plays an important role cross-culturally in vocal production and musical theory, 
perceptual evidence of the related chroma phenomenon in humans is difficult to find. Although several tests have been developed to test humans for accurate pitch chroma perception without musical training (Ross, Olson, Marks, \& Gore, 2004; Weisman, Balkwill, et al., 2012), most humans do fairly poorly at these tasks. In addition, when asked to rate the similarity between two notes, human participants tend to focus on differences in pitch height rather than chroma, especially nonmusicians (Allen, 1967; Krumhansl \& Shepard, 1979). However, if participants were given options only with similar chroma, not pitch height (e.g., rating the similarity of notes that were either an octave apart or almost an octave apart), only then would they attend to pitch chroma (Kallman, 1982). A study with rats showed that the rats generalize across octaves (Blackwell \& Schlosberg, 1943), but it was later criticized for not controlling for harmonics that would have included octave information (Burns, 1999). One study showed evidence that rhesus monkeys (Macaca mulatta) attend to octaves when recognizing melodies (Wright, Rivera, Hulse, Shyan, \& Neiworth, 2000). Another study showed that European starlings (Sturnus vulgaris) do not show octave equivalence (Cynx, 1993). However, the design used was much like the human studies that failed to show chroma perception (Allen, 1967; Kallman, 1982; Krumhansl \& Shepard, 1979). Later, my colleagues and I showed that, indeed, humans also fail at the task that was used with this species, also relying on pitch height rather than pitch chroma (Hoeschele, Weisman, \& Sturdy, 2012), much like humans in previous studies. As a response to this literature, we designed our own octave perception task using a similar three-range operant conditioning task to the ones used to test for pitch height by Weisman and colleagues. Here we trained participants to differentially respond to notes presented in one octave and then tested them in a different octave to avoid pitch height effects as much as possible. We were successfully able to

Figure 1. The pitch interval with the greatest harmonic overlap (outside of unison) is the octave. Here we demonstrate this with an example using $220 \mathrm{~Hz}(\mathrm{~A} 3)$ and $440 \mathrm{~Hz}(\mathrm{~A} 4)$. The upper portion of the figure shows the fundamental (F0) and first eight harmonics (F1-F8) of both $220 \mathrm{~Hz}$ and $440 \mathrm{~Hz}$ side by side. Below, the fundamental frequencies are displayed on their own as sinewaves to show the simplicity of the ratio between them that allows them to be heard as one sound by a listener as they pulse in time with each other.

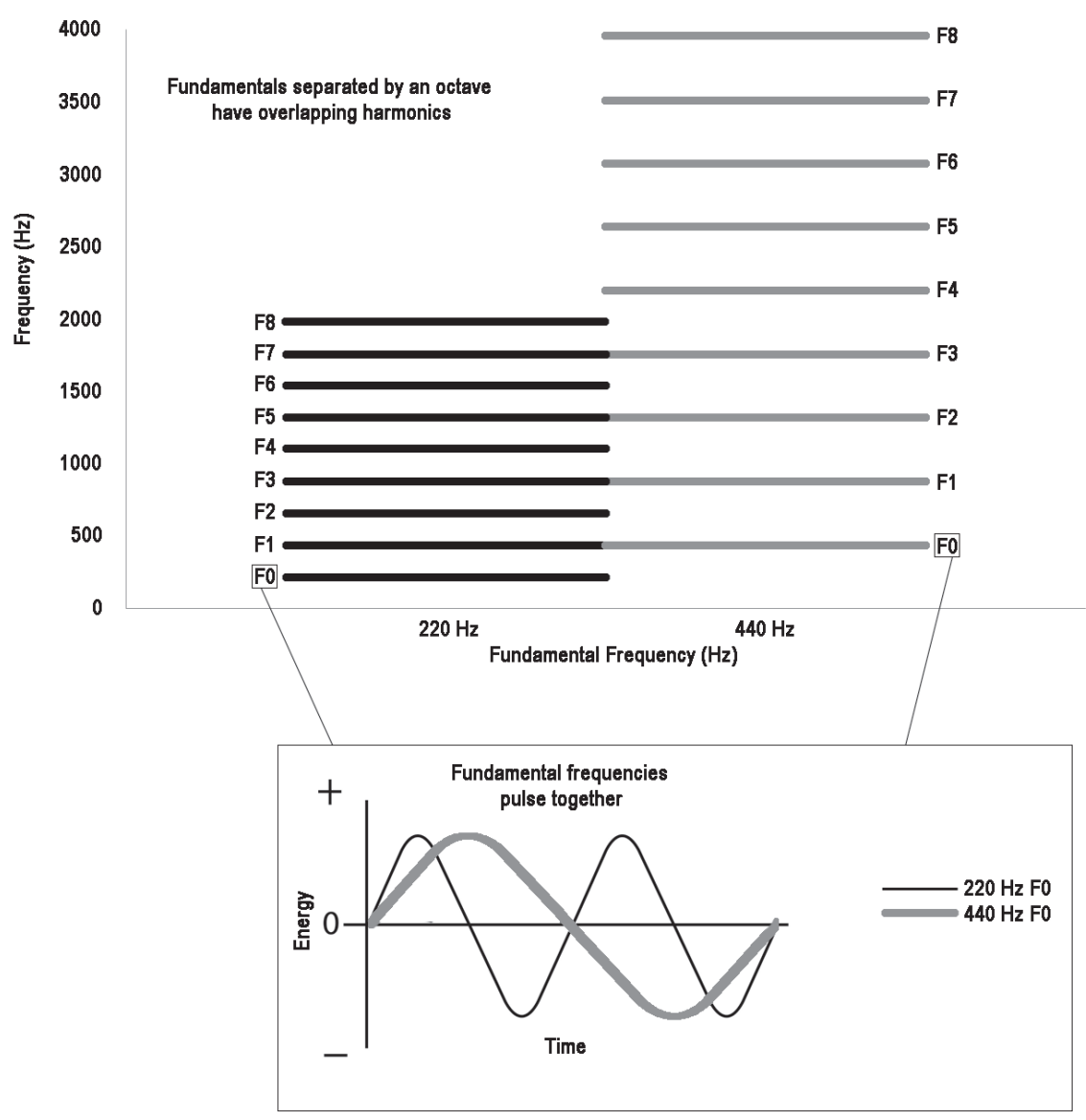


show attention to chroma by even nonmusician humans in this task (Hoeschele, Weisman, et al., 2012). A followup study with chickadees (Poecile atricapillus) showed that this songbird did not appear to attend to chroma in this same task (Hoeschele, Weisman, Guillette, Hahn, \& Sturdy, 2013). Thus, to date, only the more closely related rhesus monkey has been shown in a perceptual task to attend to the octave as humans do, but little work has been conducted on this topic.

As humans readily show octave generalization in their vocal production, it may make more sense to study the vocal production of other species. There is some evidence that other species also attend to harmonic structure when producing pitch. A dolphin (Tursiops truncatus) trained to imitate sounds was able to octave transpose sound outside her preferred range (Richards, Wolz, \& Herman, 1984). In addition, both hermit thrushes (Catharus guttatus; Doolittle et al., 2014) and musician wrens (Cyphorhinus arada; Doolittle \& Brumm, 2013) tend to sing intervals with small integer ratios, including the octave ratio of 1:2 and other ratios found within the harmonic series such as 2:3 (perfect fifth). A study with great tits (Parus major) showed that male dominance is correlated with their ability to produce simple ratios in their song (Richner, 2016). Another study with a mosquito species (Aedes aegypti) showed that males and females matched the harmonics of their flight tones to perform a courtship duet in a simple ratio of 2:3 (Cator et al., 2009). This suggests that the simple harmonic relation between a note and its octave, but also between a note and other harmonics, may contribute to how some animals produce acoustic signals. Although very few species have been shown to use simple ratios to date, it is unclear yet whether the use of simple ratios is uncommon or simply undiscovered. Further study of the natural vocalizations and imitation abilities of other species may thus help us answer whether the octave and other simple integer ratios is important to other species. Perhaps imitating species with different vocal ranges, like humans, are especially likely to attend to the octave relationship.

\section{Relative Pitch}

\section{What Is Relative Pitch?}

Except for people with musical absolute pitch, our absolute pitch abilities are not fine enough to assess the more subtle differences among notes in music. When hearing an A note followed by a D note, the majority of people would not be able to identify the notes they are hearing but instead could tell you the direction of pitch change (up or down; McDermott, Keebler, Micheyl, \& Oxenham, 2010), and people can be easily trained (normally through musical training) to identify the size of the interval (e.g., perfect fourth). This comparison of pitches is known as relative pitch. Comparing the pitches of notes allows us to recognize a tune, such as "Happy Birthday," even when it begins on a different note.

Although relative pitch is thought to be quite critical to the human musical capacity, it seems on the surface to be somewhat limited in other species. Songbird species that were trained to identify note patterns of two or more notes (such as patterns that were ascending rather than descending in frequency) tended to rely on the absolute frequencies and their positions within the pattern to learn the task (Hulse \& Cynx, 1985, 1986; Hulse, Cynx, \& Humpal, 1984; MacDougall-Shackleton \& Hulse, 1996; Njegovan \& Weisman, 1997; Page et al., 1989; Weisman, Njegovan, \& Ito, 1994). When presented with new patterns that followed the same relative pitch rule but at a novel absolute pitch, the birds needed to be retrained to respond appropriately if the notes fell outside of the training range (Hulse \& Cynx, 1985). However, the birds did use the relative pitch information to some degree, by applying a relative rule to transpositions that were within the training range (Hulse \& Cynx, 1985) and, for example, learning an absolute pitch task when they were provided with additional relative pitch information more quickly than the same task without relative pitch information (Njegovan \& Weisman, 1997). Similar work with a dolphin showed that the dolphin was able to learn a relative pitch rule and generalize this rule to novel stimuli with different absolute pitches after extensive training (Ralston \& Herman, 1995).

The work just outlined might suggest that relative pitch information is not normally encoded by nonhuman animals. However, additional studies make this conclusion unlikely. For example, by making sure that absolute and relative pitch were not in conflict with one another, researchers showed that starlings would encode both absolute and relative pitch in ascending and descending note patterns (MacDougall-Shackleton \& Hulse, 1996). In addition, other work suggests that relative pitch is used quite readily by nonhuman species with other types of stimuli. When notes are presented simultaneously, rather than sequentially, several mammalian and avian species have been shown to be able to learn relative pitch rules (Brooks \& Cook, 2010; Hoeschele, Guillette, \& Sturdy, 2012; Hulse et al., 1995; Izumi, 2000; Watanabe, Uozumi, $\&$ Tanaka, 2005). It is unclear why this is the case, but 
there are a couple possible interpretations of this data: First, presenting the notes simultaneously means that the animals can compare the fundamental frequencies of tones without needing to rely on auditory memory. Second, the animals are not attending to pitch in isolation but are attending to some other properties of the sound such as timbre, spectral shape (see Patel, in this issue), or some other "Gestalt"-like aspect of the sounds. In humans, identifying individual notes may allow us to identify the vocal part of one individual in a group. In contrast, birds can produce more than one frequency simultaneously with their vocal apparatus, the syrinx (Suthers, 1990). For avian species it therefore may make sense that they are evaluating the sound as a whole rather than attending to the individual pitches within a chord. In fact, in a follow-up study with humans and chickadees, we found that chickadees did not respond to novel timbres based on the pitch information with which they had been trained (Hoeschele, Cook, Guillette, Hahn, $\&$ Sturdy, 2014). Similar results have also been found in starlings (Bregman, Patel, \& Gentner, 2016).

However, relative pitch can be important in species other than humans in a more biological context than the aforementioned asks. For example, black-capped chickadees have a song that consists of two whistle-like notes that are separated by a relative pitch interval. This relative pitch interval is important to the birds, as it is more accurately produced by dominant males (Christie, Mennill, \& Ratcliffe, 2004). In the field, birds will respond more readily to intervals that mimic the one found in their song. In the lab, when tested for relative pitch abilities with sinewave tones, they did not perform very well (Njegovan \& Weisman, 1997). However, when presented with notes from their own song, and, to a lesser extent, sinewave tones mimicking the relative pitch relationship of these notes, they showed much more rapid acquisition of the task (Hoeschele, Guillette, et al., 2012). This is an example that reminds us that how we test animals can greatly affect the results. A similar result was found in European starlings: They also did not do well on traditional laboratory relative pitch tasks but readily recognized transposed versions of their own song (Bregman, Patel, \& Gentner, 2012). However, it is likely that they were not directly using pitch in this task but spectral shape information from which both pitch and timbre are derived (Bregman et al., 2016).

\section{Grouping and ConsonancelDissonance}

Similar to absolute pitch, relative pitch can be evaluated in several ways. In some cases, simply the direction of pitch change is important. In stress-timed languages, including English and German, listeners often attend primarily to relative pitch to identify stress (e.g., Kohler, 2012). In tonal languages, such as Mandarin and Vietnamese, the direction of pitch change within a word can change its meaning (Kim et al., 2016). It has recently been shown that both zebra finches (Spierings \& ten Cate, 2014) and budgerigars (Hoeschele \& Fitch, 2016) also attend to pitch information when identifying stress patterns in human speech.

Relative pitch may be used more generally to group stimuli. For example, when humans hear a repeating pattern of two notes that differ only in frequency, they will group the notes so that the note higher in pitch comes first. This is part of what's known as the iambictrochaic law (Bolton, 1894). There is evidence that, at least as far as pitch is concerned, the iambic-trochaic law applies to several other species, including rats (de la Mora, Nespor, \& Toro, 2013) and zebra finches (Spierings, Hubert, \& ten Cate, in press) as well. Because other species group patterns based on pitch alone, similar to humans, it appears that nonhuman animals are listening to and processing relative pitch information.

In other cases, the size of an interval is important. Depending on the size of an interval in music, it may induce different emotions (Bowling et al., 2010; Curtis \& Bharucha, 2010). The perfect intervals found in Western music are also very commonly found in other cultures and tend to be viewed as the most consonant or pleasing intervals (Burns, 1999). These intervals have simple ratios of 1:2, 2:3, and 3:4, in order from most common (octave) to least common (perfect fifth followed by perfect fourth). Notes separated by simple ratios have many overlapping harmonics, and thus they can be perceived as a single sound instead of two. Intervals with simple ratios are often identified as consonant, or pleasing. Complex ratios with little harmonic overlap are often identified as dissonant, or displeasing. Consonant sounds tend to blend together as one, whereas dissonant sounds grate against each other. However, consonance and dissonance are assessed differently across cultures and time (Carterette \& Kendall, 1999), thus there is no objective measure of consonance and dissonance. Although some cross-cultural studies have shown core similarities in the perception of consonance across cultures despite some differences (N. D. Cook, 2006; Fritz et al., 2009), others suggest that the perceptual similarities across cultures may be limited (McDermott, Schultz, Undurraga, \& Godoy, 2016). The issue of consonance and dissonance in music is additionally 
complicated by the fact that timbre also affects spectral structure and thus can influence which sounds are perceived as consonant and dissonant (Kameoka \& Kuriyagawa, 1969). Recent work has shown that humans attend to consonance information to distinguish intervals when presented with a piano timbre but not when presented with sinewaves that lack harmonic information. Instead, when presented with sinewaves, the relative size of the intervals determines their discriminability. Black-capped chickadees, on the contrary, only attended to the relative size of the intervals in all cases (Vilinsky et al., in prep.).

However, there is evidence that other species attend to consonance and dissonance (see also Toro \& CrespoBojorque, in this issue). For example, newly hatched chicks (Gallus gallus) were given the chance to imprint on an object that was correlated with either consonant or dissonant music. The chicks were more likely to imprint on the object that was presented together with consonant music (Chiandetti \& Vallortigara, 2011). A biological attraction to consonant sounds makes sense if you consider that a chick would normally imprint on its mother, which would be producing simple harmonic vocalizations that show similarities to consonant intervals (Bowling \& Purves, 2015). However, a similar study with cotton-top tamarins (Saguinus oedipus) showed no preference for consonant over dissonant intervals (McDermott \& Hauser, 2004). However, it is unclear from this study whether these animals attended the differences between the consonance and dissonant stimuli and simply were uninterested, or whether they did not notice a difference.

In addition, consonance and dissonance can explain the response patterns of both the mammalian and avian species that were trained to discriminate simultaneous pitch intervals. For example, pigeon and chickadee error patterns were similar to the error patterns of human subjects with errors reflecting level of similarity in consonance/dissonance (R. G. Cook \& Brooks, 2009; Hoeschele, Cook, Guillette, Brooks, \& Sturdy, 2012). In addition, both Java sparrows (Lonchura oryzivora) and Japanese monkeys (Macaca fuscata) showed generalization to novel chords with similar consonance and dissonance (Izumi, 2000; Watanabe et al., 2005). However, keep in mind that in a study where timbre was altered, the response patterns of chickadees were quite different than what was observed in humans (Hoeschele et al., 2014).

To conclude, despite the early evidence that animals pay little attention to relative pitch, relative pitch appears to be used in a variety of contexts at least in some species. Nonhuman animals also appear use relative pitch for perceptual grouping, identifying harmonic information that is similar to natural vocalization, and in other biologically relevant contexts, sometimes even in cases with pure tones where other features of sound are controlled for (e.g., Spierings et al., in press). It is possible that the humans we can test today tend to be more general relative pitch listeners, perhaps because of their exposure to instrumentation in music. We are used to many different sounding instruments playing melodies that we might originally have only been able to sing, which might strengthen the need to attend to the pitch over timbral information in stimuli. Our large cultural groups today mean that common songs, such as "Happy Birthday," do not have a set starting pitch, making relative pitch even more important for recognizing melodies. Testing for the use of relative pitch with a broad range of stimuli makes it clear that relative pitch perception is not just a human phenomenon. However, these tests are also a reminder that, although evaluating pitch in isolation has been tremendously helpful, context can change immensely what features of stimuli animals evaluate. For humans, relative pitch is highly relevant because we create harmonies not only with our voices but also with instruments that in many ways mimic the harmonic spectra of the voice. Studying animals that also produce signals that coincide with the harmonic series may be the key to understanding the biology underlying our use of relative pitch.

\section{Conclusions}

Thoroughly breaking down pitch has led to some interesting general conclusions about how humans compare to other animals. Many other animals do appear to attend to harmonic structure to identify pitch as exemplified by responses to stimuli with missing fundamental frequencies. We can thus say that animals generally do attend to pitch in harmonic signals. We can also be fairly confident in saying that many vocal learning avian species are more accurate at identifying pitch height in a signal, and they do this more readily than identifying relative pitch relationships, especially if the notes are not presented simultaneously. However, animals can pay attention to relative pitch and do so in a variety of more biologically relevant contexts. Similarly, all humans do pay attention to and encode absolute pitch at a surprisingly higher level than is normally thought. Thus, overall we have found that perceiving pitch, both in an absolute and relative manner, is common across 
the animal kingdom, but when and how it is assessed can vary.

While breaking down pitch and determining what it means to humans in order to study it in other animals, it becomes clear how little we actually know about humans directly through empirical testing. Much of what we know about humans and pitch is based on theory and verbal responses. Studying other animals has made it clear that pitch perception in humans is anything but simple. The things we take for granted, such as the perception of the octave relationship, turn out not to be easily shown even in humans.

Conducting comparative studies between humans and other species has made it clear that humans and other animals are perhaps less different than might be expected from music theory. It has made us appreciate some biological differences between humans and other animals, such as the potential importance of differences in vocal range between males and females in human octave perception and the potential relevance of musical instruments in human pitch perception. Pitched musical instruments, tools for which pitch can be altered to perform acoustic displays, have yet to be discovered in other species as far as I know.

Recent work suggests that the perceptual border of pitch and timbre is less clear than has been suggested in the human literature (see also Patel, in this issue). Rather than focusing on pitch in future research, which is defined as a percept, we should focus on features of sound and in what contexts these features are relevant, such as fundamental frequency, ratio of fundamental frequencies, and spectral information as a whole such as spectral shape. This may also change how we view pitch in humans, who may also rarely break down a signal purely into pitch and timbre as is supposed in music theory.

\section{References}

Allen, D. (1967). Octave discriminability of musical and non-musical subjects. Psychonomic Science, 7, 421-422. doi:10.3758/BF03331154

Athos, E. A., Levinson, B., Kistler, A., Zemansky, J., Bostrom, A., Freimer, N., \& Gitschier, J. (2007). Dichotomy and perceptual distortions in absolute pitch ability. Proceedings of the National Academy of Sciences of the United States of America, 104, 14795-14800. doi:10.1073/pnas.0703868104
Bachem, A. (1955). Absolute pitch. Journal of the Acoustical Society of America, 27, 1180-1185. doi:10.1121/1.1908155

Baharloo, S., Johnston, P., Service, S. K., Gitschier, J., \& Freimer, N. B. (1998). Absolute pitch: an approach for identification of genetic and nongenetic components. American Journal of Human Genetics, 62, 224-231. doi:10.1086/301704

Benetos, E., Dixon, S., Giannoulis, D., \& Kirchhoff, H. (2013). Automatic music transcription : Challenges and future directions. Journal of Intelligent Information Systems, 41, 407-434. doi:10.1007/ s10844-013-0258-3

Blackwell, H. R., \& Schlosberg, H. (1943). Octave generalization, pitch discrimination, and loudness thresholds in the white rat. Journal of Experimental Psychology, 33, 407-419. doi:10.1037/h0057863

Bolton, T. J. (1894). Rhythm. American Journal of Psychology, 6, 145-238. doi:10.2307/1410948

Bowling, D. L., Gill, K., Choi, J. D., Prinz, J., \& Purves, D. (2010). Major and minor music compared to excited and subdued speech. The Journal of the Acoustical Society of America, 127, 491-503. doi:10.1121/1.3268504

Bowling, D. L., \& Purves, D. (2015). A biological rationale for musical scales. Proceedings of the National Academy of Sciences, 112, 11155-11160. doi:10.1371/journal.pone.0008144

Bregman, M. R., Patel, A. D., \& Gentner, T. Q. (2012). Stimulus-dependent flexibility in non-human auditory pitch processing. Cognition, 122, 51-60. doi:10.1016/j.cognition.2011.08.008

Bregman, M. R., Patel, A. D., \& Gentner, T. Q. (2016). Songbirds use spectral shape, not pitch, for sound pattern recognition. Proceedings of the National Academy of Sciences, 113(6), 1-6. doi:10.1073/ pnas. 1515380113

Brooks, D. I., \& Cook, R. G. (2010). Chord discrimination by pigeons. Music Perception, 27, 183-196. doi:10.1525/MP.2010.27.3.183 
Brown, S., \& Jordania, J. (2011). Universals in the world's musics. Psychology of Music, 41, 229-248. doi:10.1177/0305735611425896

Burgoyne, J. A., Wild, J., \& Fijinaga, I. (2011). An Expert Ground-Truth Set for Audio Chord Recognition and Music Analysis. In Proceedings of the 12th International Society for Music Information Retrieval Conference. Miami (Florida), USA. October 24-28. pp. 633-638.

Burns, E. M. (1981). Circularity in relative pitch judgments for inharmonic complex tones: The Shepard demonstration revisited, again. Perception \& Psychophysics, 30, 467-472. doi:10.3758/ BF03204843. Retrieved from http://www.ncbi.nlm. nih.gov/pubmed/7329764

Burns, E. M. (1999). Intervals, scales, and tuning. In D. Deutsch (Ed.), The psychology of music (2nd ed., pp. 215-264). San Diego, CA: Academic Press. doi:10.1016/b978-012213564-4/50008-1

Carroll, J. B. (1975). Speed and accuracy of absolute pitch judgments: Some latter-day results. Princeton, NJ: Educational Testing Service. doi:10.1002/j.2333-8504.1975.tb01075.x

Carterette, E. C., \& Kendall, R. A. (1999). Comparative music perception and cognition. In D. Deutsch (Ed.), The psychology of musicca (2nd ed., pp. 725-791). San Diego, CA: Academic Press. doi:10.1016/B978-012213564-4/50019-6

Cator, L. J., Arthur, B. J., Harrington, L. C., \& Hoy, R. R. (2009). Harmonic convergence in the love songs of the dengue vector mosquito. Science, 323, 10771079. doi:10.1126/science.1166541

Chiandetti, C., \& Vallortigara, G. (2011). Chicks like consonant music. Psychological Science, 22, 1270 1273. doi:10.1177/0956797611418244

Christie, P. J., Mennill, D. J., \& Ratcliffe, L. M. (2004). Pitch shifts and song structure indicate male quality in the dawn chorus of black-capped chickadees. Behavioral Ecology and Sociobiology, 55, 341-348. doi:10.1007/s00265-003-0711-3
Cook, N. D. (2006). The psychophysics of harmony perception: Harmony is a three-tone phenomenon. Empirical Musicology Review, 1, 106-126.

Cook, R. G., \& Brooks, D. I. (2009). Generalized auditory same-different discrimination by pigeons. Journal of Experimental Psychology. Animal Behavior Processes, 35, 108-115. doi:10.1037/ a0012621

Curtis, M. E., \& Bharucha, J. J. (2010). The minor third communicates sadness in speech, mirroring its use in music. Emotion, 10, 335-348. doi:10.1037/ a0017928

Cynx, J. (1993). Auditory frequency generalization and a failure to find octave generalization in a songbird, the European starling (Sturnus vulgaris). Journal of Comparative Psychology, 107, 140-146. doi:10.1037/0735-7036.107.2.140

Cynx, J., \& Shapiro, M. (1986). Perception of missing fundamental by a species of songbird (Sturnus vulgaris). Journal of Comparative Psychology, 100, 356-360. Retrieved from http://www.ncbi.nlm.nih.gov/pubmed/3802780. doi:10.1037/0735-7036.100.4.356

de la Mora, D. M., Nespor, M., \& Toro, J. M. (2013). Do humans and nonhuman animals share the grouping principles of the iambic-trochaic law? Attention, Perception \& Psychophysics, 75, 92-100. doi:10.3758/s13414-012-0371-3

Deutsch, D. (1991). The Tritone Paradox : An influence of language on music perception. Music Perception, 8, 335-347. doi:10.2307/40285517

Deutsch, D., \& Henthorn, T. (2004). Absolute pitch, speech, and tone language: Some experiments and a proposed framework. Music Perception, 21, 339-356. doi:10.1525/mp.2004.21.3.339

Doolittle, E., \& Brumm, H. (2013). O Canto do Uirapuru: Consonant intervals and patterns in the song of the musician wren. Journal of Interdisciplinary Music Studies, 6, 55-85. doi:10.4407/jims.2013.10.003 
Doolittle, E. L., Gingras, B., Endres, D. M., \& Fitch, W. T. (2014). Overtone-based pitch selection in hermit thrush song: Unexpected convergence with scale construction in human music. Proceedings of the National Academy of Sciences, 111, 16616-16621. doi:10.1073/pnas.1406023111

Doupe, J., \& Kuhl, P. K. (1999). Birdsong and human speech: Common themes and mechanisms. Annual Review of Neuroscience, 22, 567-631. doi:10.1146/ annurev.neuro.22.1.567

Dowling, W. J., \& Harwood, D. L. (1986). Music cognition. Cognitive processing. Orlando, FL: Academic Press. Retrieved from http://www.ncbi. nlm.nih.gov/pubmed/20374926

Filippi, P. (2016). Emotional and interactional prosody across animal communication systems : A comparative approach to the emergence of language. Frontiers in Psychology, 7, 1393. doi:10.3389/fpsyg.2016.01393

Fitch, W. T. (2006). On the biology and evolution of music. Music Perception, 24, 85-88. doi:10.1525/ mp.2006.24.1.85

Fitch, W. T., \& Hauser, M. D. (2003). Unpacking "honesty": Vertebrate vocal production and evolution of acoustic signals. Springer Handbook of Auditory Research, 16, 65-137. doi:10.1007/0-387-22762-8_3

Friedrich, A., Zentall, T., \& Weisman, R. (2007). Absolute pitch: Frequency-range discriminations in pigeons (Columba livia): Comparisons with zebra finches (Taeniopygia guttata) and humans (Homo sapiens). Journal of Comparative Psychology, 121, 95-105. doi:10.1037/0735-7036.121.1.95

Fritz, T., Jentschke, S., Gosselin, N., Sammler, D., Peretz, I., Turner, R., ... Koelsch, S. (2009). Report universal recognition of three basic emotions in music. Current Biology, 19, 573-576. doi:10.1016/j. cub.2009.02.058

Halpern, A. R., \& Müllensiefen, D. (2008). Effects of timbre and tempo change on memory for music. The Quarterly Journal of Experimental Psychology, 61, 1371-1384. doi:10.1080/17470210701508038
Heffner, H., \& Whitfield, I. C. (1976). Perception of the missing fundamental by cats. The Journal of the Acoustical Society of America, 59, 915-919. doi:10.1121/1.380951

Hoeschele, M., Cook, R. G., Guillette, L. M., Brooks, D. I., \& Sturdy, C. B. (2012). Black-capped chickadee (Poecile atricapillus) and human (Homo sapiens) chord discrimination. Journal of Comparative Psychology, 126, 57-67. doi:10.1037/ a0024627

Hoeschele, M., Cook, R. G., Guillette, L. M., Hahn, A. H., \& Sturdy, C. B. (2014). Timbre influences chord discrimination in black-capped chickadees (Poecile atricapillus) but not humans (Homo Sapiens). Journal of Comparative Psychology, 128, 387-401. doi:10.1037/a0037159

Hoeschele, M., \& Fitch, W. T. (2016). Phonological perception by birds: Budgerigars can perceive lexical stress. Animal Cognition, 19, 643-654. doi:10.1007/s10071-016-0968-3

Hoeschele, M., Guillette, L. M., \& Sturdy, C. B. (2012). Biological relevance of acoustic signal affects discrimination performance in a songbird. Animal Cognition, 15, 677-688. doi:10.1007/ s10071-012-0496-8

Hoeschele, M., Merchant, H., Kikuchi, Y., Hattori, Y., $\&$ ten Cate, C. (2015). Searching for the origins of musicality across species. Philosophical Transactions of the Royal Society B, 370, 20140094. doi:10.1098/ rstb.2014.0094

Hoeschele, M., Weisman, R. G., Guillette, L. M., Hahn, A. H., \& Sturdy, C. B. (2013). Chickadees fail standardized operant tests for octave equivalence. Animal Cognition, 16, 599-609. doi:10.1007/ s10071-013-0597-Z

Hoeschele, M., Weisman, R. G., \& Sturdy, C. B. (2012). Pitch chroma discrimination, generalization, and transfer tests of octave equivalence in humans. Attention, Perception \& Psychophysics, 74, 17421760. doi:10.3758/s13414-012-0364-2 
Hulse, S. H., Bernard, D. J., \& Braaten, R. E. (1995). Auditory discrimination of chord-based spectrial structures by European starlings (Sturnus vulgaris). Journal of Experimental Psychology: General, 124, 409-423. doi:10.1037/0096-3445.124.4.409

Hulse, S. H., \& Cynx, J. (1985). Relative pitch perception is constrained by absolute pitch in songbirds (Mimus, Molothrus, and Sturnus). Journal of Comparative Psychology, 99, 176-196. doi:10.1037/0735-7036.99.2.176

Hulse, S. H., \& Cynx, J. (1986). Interval and contour in serial pitch perception by a passerine bird, the European starling (Sturnus vulgaris). Journal of Comparative Psychology, 100, 215-228. doi:10.1037/0735-7036.100.3.215

Hulse, S. H., Cynx, J., \& Humpal, J. (1984). Absolute and relative pitch discrimination in serial pitch perception by birds. Journal of Experimental Psychology: General, 113, 38-54. doi:10.1037/0096-3445.113.1.38

Izumi, A. (2000). Japanese monkeys perceive sensory consonance of chords. The Journal of the Acoustical Society of America, 108, 3073-3078. doi:10.1121/1.1323461

Kallman, H. J. (1982). Octave equivalence as measured by similarity ratings. Perception \& Psychophysics, 32, 37-49. doi:10.3758/BF03204867

Kameoka, A., \& Kuriyagawa, M. (1969). Consonance Theory Part II: Consonance of Complex Tones and Its Calculation Method. Journal of the Acoustical Society of America, 45, 1460-1469. doi:10.1121/1.1911624

Kim, M., Vu, K. L., Strybel, T. Z., Ngo, M. K. I. M., Vu, P. L., \& Strybel, T. Z. (2016). Effects of music and tonal language experience on relative pitch performance. The American Journal of Psychology, 129, 125-134. doi:10.5406/amerjpsyc.129.2.0125

Klapuri, A., \& Davy, M. (2006). Signal processing methods for music transcription. New York, NY: Springer. doi:10.1007/0-387-32845-9
Kohler, K. J. (2012). The perception of lexical stress in German: Effects of segmental duration and vowel quality in different prosodic patterns. Phonetica, 69, 68-93. doi:10.1159/000342126

Krumhansl, C. L., \& Shepard, R. N. (1979). Quantification of the hierarchy of tonal functions within a diatonic context. Journal of Experimental Psychology. Human Perception and Performance, 5, 579-594. doi:10.1037/0096-1523.5.4.579

Lange, K., \& Czernochowski, D. (2013). Acta Psychologica Does this sound familiar? Effects of timbre change on episodic retrieval of novel melodies. Acta Psychologica, 143, 136-145. doi:10.1016/j. actpsy.2013.03.003

Latinus, M., \& Taylor, M. J. (2012). Discriminating male and female voices : Differentiating pitch and gender. Brain Topography, 25, 194-204. doi:10.1007/ s10548-011-0207-9

Lau, B. K., \& Werner, L. A. (2012). Perception of missing fundamental pitch by 3- and 4-month-old human infants. The Journal of the Acoustical Society of America, 132, 3874-3882. doi:10.1121/1.4763991

Lee, T. T. Y., Charrier, I., Bloomfield, L. L., Weisman, R. G., \& Sturdy, C. B. (2006). Frequency-range discriminations and absolute pitch in black-capped chickadees (Poecile atricapillus), mountain chickadees (Poecile gambeli), and zebra finches (Taeniopygia guttata). Journal of Comparative Psychology, 120, 217-228. doi:10.1037/0735-7036.120.3.217

Levitin, D. J. (1994). Absolute memory for musical pitch: Evidence from the production of learned melodies. Perception \& Psychophysics, 56, 414-423. doi:10.3758/BF03206733

Lockhead, G. R., \& Byrd, R. (1981). Practically perfect pitch. Journal of the Acoustical Society of America, 70, 387-389. doi:10.1121/1.386773

MacDougall-Shackleton, S. A., \& Hulse, S. H. (1996). Concurrent absolute and relative pitch processing by European starlings (Sturnus vulgaris). Journal of Comparative Psychology, 110, 139-146. doi:10.1037//0735-7036.110.2.139 
McDermott, J. H., Keebler, M. V, Micheyl, C., \& Oxenham, A. J. (2010). Musical intervals and relative pitch: Frequency resolution, not interval resolution, is special. Journal of the Acoustical Society of America, 128, 1943-1951. doi:10.1121/1.3478785

McDermott, J. H., Schultz, A. F., Undurraga, E. A., \& Godoy, R. A. (2016). Indifference to dissonance in native Amazonians reveals cultural variation in music perception. Nature, 25, 21-25. doi:10.1038/ nature 18635

McDermott, J., \& Hauser, M. (2004). Are consonant intervals music to their ears? Spontaneous acoustic preferences in a nonhuman primate. Cognition, 94 , B11-B21. doi:10.1016/j.cognition.2004.04.004

Miyazaki, K. (1988). Musical pitch identification by absolute pitch possessors. Perception \& Psychophysics, 44, 501-512. doi:10.3758/ BF03207484

Miyazaki, K. (1989). Absolute pitch identification : Effects of timbre and pitch region. Music Perception, 7, 1-14. doi:10.2307/40285445

Njegovan, M., \& Weisman, R. (1997). Pitch discrimination in field- and isolation-reared black-capped chickadees (Parus atricapillus). Journal of Comparative Psychology, 111, 294-301. doi:10.1037/0735-7036.111.3.294

Page, S. C., Hulse, S. H., \& Cynx, J. (1989). Relative pitch perception in the European starling (Sturnus vulgaris): Further evidence for an elusive phenomenon. Journal of Experimental Psychology. Animal Behavior Processes, 15, 137-146. doi:10.1037/0097-7403.15.2.137

Patel, A. D. (2003). Language, music, syntax and the brain. Nature Neuroscience, 6, 674-681. doi:10.1038/nn1082

Ralston, J. V., \& Herman, L. M. (1995). Perception and generalization of frequency contours by a bottlenose dolphin (Tursiops truncatus). Journal of Comparative Psychology, 109, 268-277. doi:10.1037/0735-7036.109.3.268
Richards, D. G., Wolz, J. P., \& Herman, L. M. (1984). Vocal mimicry of computer-generated sounds and vocal labeling of objects by a bottlenosed dolphin, tursiops truncatus. Journal of Comparative Psychology, 98, 10-28. doi:10.1037/0735-7036.98.1.10

Richner, H. (2016). Interval singing links to phenotypic quality in a songbird. Proceedings of the National Academy of Sciences, 113, 12763-12767. doi:10.1073/pnas. 1610062113

Ross, D. A., Olson, I. R., Marks, L. E., \& Gore, J. C. (2004). A nonmusical paradigm for identifying absolute pitch possessors. Journal of the Acoustical Society of America, 116, 1793-1799. doi:10.1121/1.1758973

Rothenberg, D., Roeske, T. C., Voss, H. U., Naguib, M., \& Tchernichovski, O. (2014). Investigation of musicality in birdsong. Hearing Research, 308, 71-83. doi:10.1016/j.heares.2013.08.016

Schellenberg, E., Stanlinski, S., \& Marks, B. M. (2013). Memory for surface features of unfamiliar melodies: Independent effects of changes in pitch and tempo. Psychological Research, 78, 84-95. doi:10.1007/s00426-013-0483-y

Schellenberg, E. G., \& Trehub, S. E. (2003). Good pitch memory is widespread. Psychological Science, 14, 262-266. doi:10.1111/1467-9280.03432

Smith, N. A., \& Schmuckler, M. A. (2008). Dial A440 for absolute pitch: Absolute pitch memory by non-absolute pitch possessors. Journal of the Acoustical Society of America, 123(4), 77-84. doi:10.1121/1.2896106

Spierings, M., Hubert, J., \& ten Cate, C. (2017). Selective auditory grouping by zebra finchs: Testing the Iambic-Trochaic law. Animal Cognition. doi:10.1007/s10071-017-1089-3

Spierings, M. J., \& ten Cate, C. (2014). Zebra finches are sensitive to prosodic features of human speech. Proceedings of the Royal Society B: Biological Sciences, 281, 20140480. doi:10.1098/rspb.2014.0480 
Suthers, R. A. (1990). Contributions to birdsong from the left and right sides of the intact syrinx. Nature, 347, 473-477. doi:10.1038/347473a0

Takeuchi, A. H., \& Hulse, S. H. (1993). Absolute pitch. Psychological Bulletin, 113, 345-361. doi:10.1037/0033-2909.113.2.345

Titze, I. R. (2000). Principles of voice production. Iowa City, IA: National Center for Voice and Speech.

Tomlinson, R. W., \& Schwarz, D. W. (1988). Perception of the missing fundamental in nonhuman primates. Journal of the Acoustical Society of America, 84, 560-565. doi:10.1121/1.396833

Vilinsky, L., Hahn, A. H., Hoang, J., Hoeschele, M., Guillette, L. M., \& Sturdy, C. B. (in prep.). Timbre influences the perception of consonance and dissonance in humans (Homo sapiens) but not black-capped chickadees (Poecile atricapillus). Manuscript in preparation.

Warker, J. A., \& Halpern, A. R. (2005). Musical stem completion: Humming that note. The American Journal of Psychology, 118, 567-585.

Watanabe, S., Uozumi, M., \& Tanaka, N. (2005). Discrimination of consonance and dissonance in Java sparrows. Behavioural Processes, 70, 203-208. doi:10.1016/j.beproc.2005.06.001

Weisman, R. G., Balkwill, L.-L., Hoeschele, M., Moscicki, M. K., Bloomfield, L. L., \& Sturdy, C. B. (2010). Absolute pitch in boreal chickadees and humans: Exceptions that test a phylogenetic rule. Learning and Motivation, 41, 156-173. doi:10.1016/j.1mot.2010.04.002
Weisman, R. G., Balkwill, L.-L., Hoeschele, M., Moscicki, M. K., \& Sturdy, C. B. (2012). Identifying absolute pitch possessors without using a notenaming task. Psychomusicology: Music, Mind, and Brain, 22, 46-54. doi:10.1037/a0028940

Weisman, R. G., Mewhort, D. J. K., Hoeschele, M., \& Sturdy, C. B. (2012). New perspectives on absolute pitch in birds and mammals. In E. A. Wasserman \& T. R. Zentall (Eds.), The Oxford handbook of comparative cognition (2nd ed., pp. 67-82). New York, NY: Oxford University Press. Retrieved from http://www.oxfordhandbooks.com/ view/10.1093/oxfordhb/9780195392661.001.0001/ oxfordhb-9780195392661-e-005

Weisman, R. G., Njegovan, M. G., \& Ito, S. (1994). Frequency ratio discrimintion by zebra finches (Taeniopygia guttata) and humans (Homo sapiens). Journal of Comparative Psychology, 108, 363-372. doi:10.1037/0735-7036.108.4.363

Weiss, M. W., Vanzella, P., Schellenberg, E. G., \& Trehub, S. E. (2015). Pianists exhibit enhanced memory for vocal melodies but not piano melodies. The Quarterly Journal of Experimental Psychology, 68(5), 37-41. doi:10.1080/17470218.2015.1020818

Wright, A. A., Rivera, J. J., Hulse, S. H., Shyan, M., \& Neiworth, J. J. (2000). Music perception and octave generalization in rhesus monkeys. Journal of Experimental Psychology. General, 129, 291-307. doi:10.10371/0096-3445.129.3.291 